Apidologie, 1982, 13 (3), 241-246.

\title{
ERFAHRUNGEN MIT EINEM BIENENFLUGRAUM SPONTANE KÖNIGINNENAUFZUCHT UND LEBENSDAUERANALYSEN
}

\author{
Ulrike ROTHE und Werner NACHTIGALL \\ Zoologisches Institut der Universität des Saarlandes, Fachbereich 16 \\ Fachrichtung 4, D-6600 Saarbrücken (B.R.D.)
}

\section{ZUSAMMENFASSUNG}

Es wird die nicht induzierte Nachzucht von Königinnen im Flugraum beschrieben. Das mittlere Lebensalter von Arbeiterinnen im Flugraum lag bei drei Analysen im Mittel bei 39.4 Tagen.

\section{EINLEITUNG}

Um ganzjährig Bienen für stoffwechselphysiologische Untersuchungen aus einem brutaktiven Volk entnehmen zu können, wurde an unserem Institut ein kleiner Bienenflugraum zur Haltung von einem Bienenvolk eingerichtet. Drei Jahre hat sich dieser Modus der Bienenhaltung bewährt und zufriedenstellende Erfolge gezeigt. Das erste Volk überlebte als Versuchsvolk im Flugraum (FR) mehr als zwei Jahre. Ein 7Waben-Volk der Rasse Apis mellifica carnica wurde im Dezember 1978 in den FR gestellt. Die Haltungsbedingungen sind an anderer Stelle beschrieben (ROTHE u. Nachtigall, 1980). Erwähnt sei nur die Proteinnahrung : Es wurde tiefgefroren (- 30 Grad Celsius) gelagerter Pollen gemahlen, der als Ungarischer Blütenpollen nach der Lieferung direkt in luftdichte Plastikbeutel eingeschweisst und tiefgefroren wurde. Das FR-Volk wurde nie durch Brutwaben aus dem Freiland verstärkt. Es wurden lediglich einige Honigwaben beigehängt und Randwaben mit Wasser gefüllt. Während der FRHaltung umfasste das Brutnest eineinhalb bis drei Waben. Zur Zeit des Umweiselungversuches waren 2-3 Waben gut belagert und bebrütet; 6 Futterwaben waren von Bienen wenig belagert. Es handelte sich also um ein relativ schwaches Volk. 


\section{CHRONOLOGIE DER KÖNIGINNENNACHZUCHT IM FR}

Die Nachzucht der Königin erstreckte sich von Mai bis September des Jahres 1980.

Im Mai wird im oberen Drittel einer schwach bebrüteten Wabe spontan eine gut verhaftete Weiselzelle angelegt. Die Legetätigkeit der Königir liegt zu diesem Zeitpunkt nicht deutlich unter ihrem sonstigen Legeverhalten. Ende Juni ist diese Weiselzelle fertig gebaut, wird aber nicht bestiftet. Die Stockkönigin setzt ihre Legetätigkeit auf zwei Brutwaben fort.

Es handelte sich hier zunächst um den Versuch einer stillen Umweiselung, der natürlicherweise bei Anwesenheit der Stockkönigin zur Aufzucht von Königinnen führt. Drohnenaufzucht, die der natürlichen Königinnenaufzucht beim Schwärmen vorausgeht, ist in Flugräumen meines Wissens noch nicht beschrieben worden.

Anfang September liegt die vier Jahre alte Stockkönigin umgeben vom " Hofstaat " tot ausserhalb des Stockes (s. Abb. 1 a). Die Stockbienen sind nervös und brausen auf. Die im Mai angelegte Weiselzelle ist etwas zurückgebildet. Ihre Wabe wird nur noch als Futterwabe genutzt. Nach Verlust der Königin entstehen zwei Nachschaffungzellen aus dünnen Wachsschichten und eine kräftige Weiselzelle auf einer Nachbarwabe am oberen Wabenrand (s. Abb. $1 b, c)$. Wenige Tage später sind die zarten, leeren Weiselbecher zurückgebildet. Auf der Nachbarwabe werden zwei verdeckelte Weiselzellen gefunden; eine davon wurde beim Bauen von Bienen abgedeckt und so erst in diesem Stadium entdeckt. Auf einer dritten Wabe sind zwei Weiselzellen mit jungen Larven. $\mathrm{Zu}$ diesem Zeitpunkt ist die alte Stockkönigin nicht mehr auffindbar. Später werden die zarten Weiselbecher gänzlich zurückgebildet. Die verdeckelten Königinnenzellen sind intakt. Von den beiden anderen Weiselzellen auf der dritten Wabe ist eine wieder zurückgebildet, die andere ohne Brut. Dieser Zustand bleibt unverändert, bis eines Tages eine tote Jungkönigin ausserhalb des Stockes gefunden wird. Im Stock wird am selben Tag eine zweite, gut entwickelte Königin auf den Waben gefunden (s. Abb. $1 \mathrm{f}$ ). Beide Königinnen entstammen den jetzt geöffneten Weiselzellen, die als einzige verdeckelt worden waren (s. Abb. $1 e$ ). Die nachgezogene Königin wird abgefangen, um dem Volk eine begattete Königin zu überlassen.

Die oben beschriebene, spontane Nachzucht der Königin erfolgte also nach dem plötzlichen Verlust der Stockkönigin im Flugraum spontan, d.h. ohne steuernde Eingriffe. Diese Nachzuchtmethode wird neben Schwärmen und stiller Umweiselung auch im Freiland angewendet.

\section{LEBENSDAUERANALYSEN}

Für die spätere Versuchsplanung musste das mittlere Lebensalter der FR-Bienen bekannt sein. Dazu wurden drei Lebensdaueranalysen mit unterschiedlicher Tierzahl durchgeführt. 

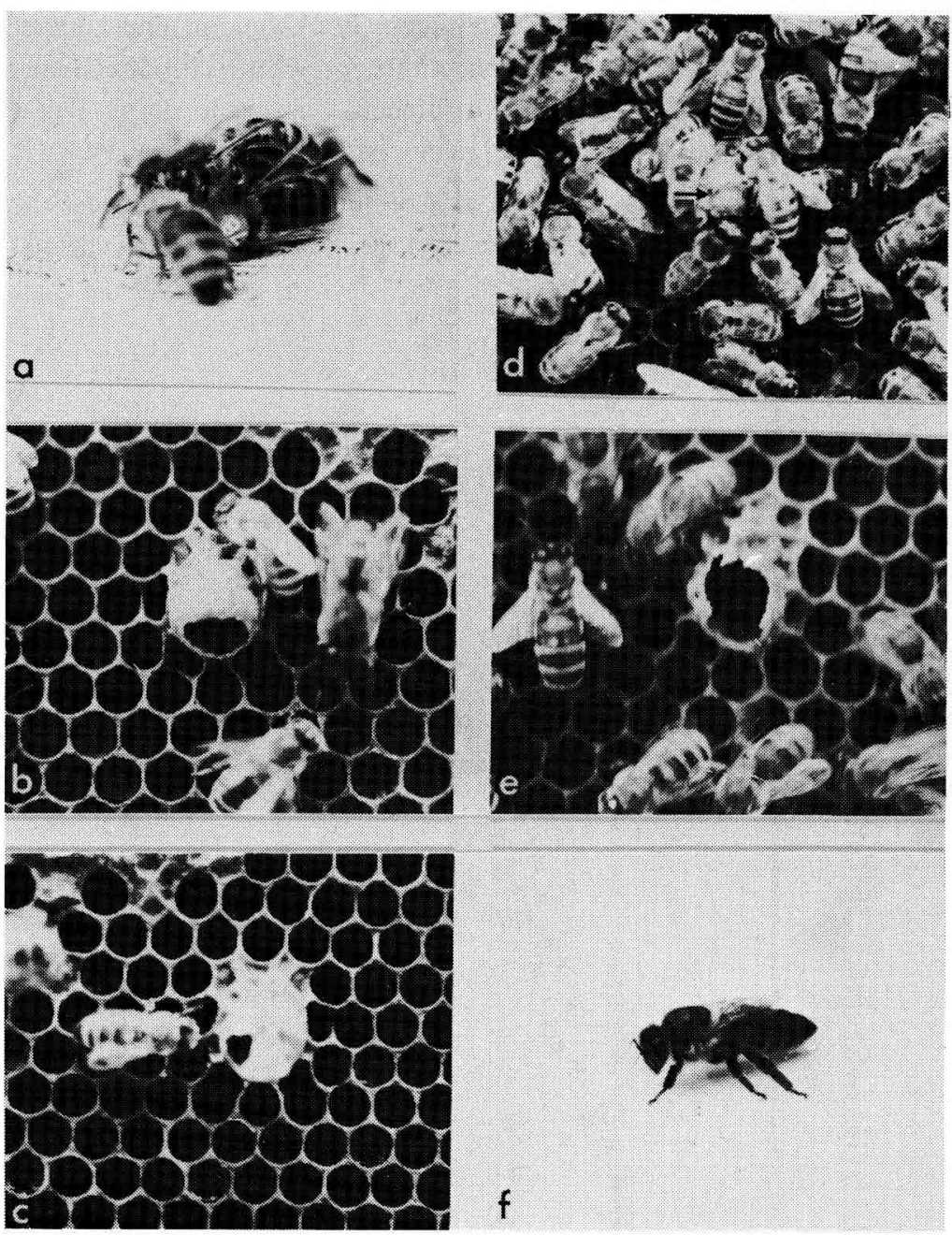

ABB. 1. - Nachzucht einer Königin nach dem Tod der Stockkönigin.

a) tote Stockkönigin.

b) eine Nachschaffungszelle.

c) diese Zelle einige Tage später.

d) diese Zelle fertig verdeckelt.

e) nach Schlüpfen der Königin.

f) FR-Königin.

FIG. 1. - Queen rearing after the death of the queen bee.
a) dead queen.
b) queen cell.
c) queen cell a few days later.
d) queen cell covered.
e) empty queen cell.
f) flight-room queen. 
Die Testzeiträume lagen jeweils bei verschiedenen Jahreszeiten. Alle markierten Arbeiterinnen entstammten jeweils von derselben Stockkönigin. Die Untersuchungen wurden immer auf die gleiche Weise durchgeführt. Frisch geschlüpfte Bienen wurden mit Markierungsschildchen numeriert. Das Bienenalter wurde durch Kontrollen im Stock und im Totenfall auf \pm einen Tag genau bestimmt.

Das Ergebnis der drei Messreihen ist in Abbildung 2 zusammengestellt. Wie aus der Kurve (s. Abb. 2) ersichtlich ist, werden etwa $50 \%$ aller Tiere älter als 40 Tage und

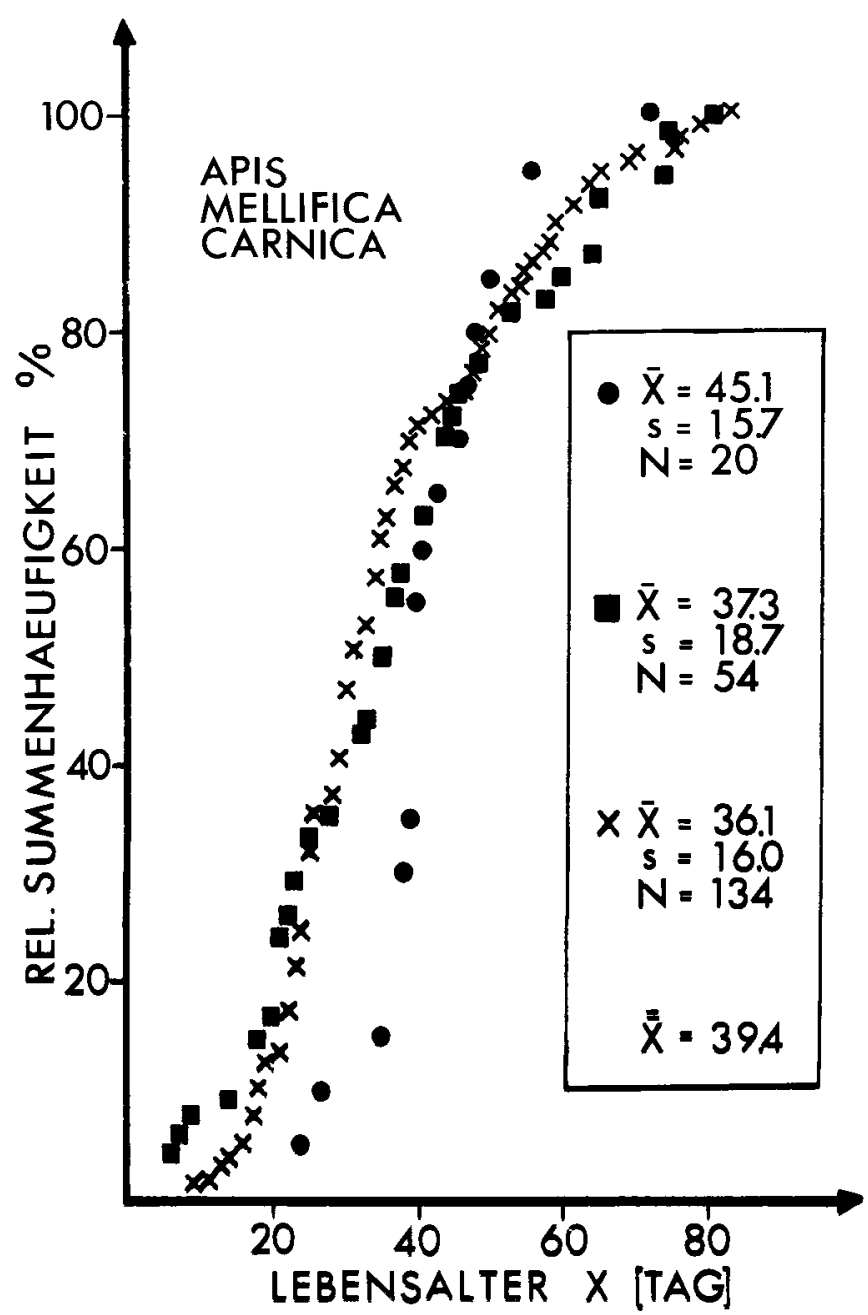

Aвв. 2. - Ergebnisse der drei Lebensdaueranalysen.

$x$-axis : life span (in days).

FIG. 2. - Results of three lifespan-analysis.

$y$-axis : relative cumulative frequency (\%). 
etwa $20 \%$ aller Tiere älter als 50 Tage. Die ältesten Tiere lebten etwa 80 Tage lang. Das relativ hohe mittlere Lebensalter von 39.4 Tagen ist erfreulich, da "gute" Versuchstiere relativ lange getestet werden können.

Die erhaltenen Werte stimmen gut mit anderen Lebensdauerdaten von Flugraumbienen überein. MeYER (1982) gibt seinen Mittelwert mit etwa 38-42 Tagen an. Das Lebensalter von freilebenden Sammlerinnen wird dagegen am häufigsten mit 45 bzw. 5-6 Wochen angegeben (v. Frisch, 1969; Grout u. RUTTNER, 1973; Peschetz, 1954; Butler, 1954; HornsmanN, 1956; Leuenberger, 1948; RAUTENBERG, 1946; RIBBANDS, 1953).

Es sind verschiedene Gründe für das relativ hohe Bienenalter im FR verglichen mit freilebenden Sommerbienen denkbar. Einerseits könnte das Fehlen von Feinden und die relativ einfache Nahrungsbeschaffung dazu beitragen. Zuckerwasser und Blütenpollen werden im FR stets ad libitum auf einer Futterstelle geboten; Nahrung ist also im Uberfluss vorhanden und leicht an einer Futterstelle zu gewinnen. Andererseits könnte das reichliche Proteinangebot der gemahlenen Pollenhöschen in Kombination mit geringer Brutaktivität die Lebzeit der Arbeiterinnen ähnlich wie bei Sommerbienen im Freiland bei stagnierendem Brutgeschäft verlängern (MAURIzıo, 1950).

Eingegangen im Juni 1982. Reçu pour publication en juin 1982.

\section{SUMMARY}

\section{SPONTANEOUS QUEEN REARING AND HONEYBEE WORKER LONGEVITY IN A FLIGHT ROOM}

A honeybee queen was kept continously for nearly two years in a flight-room (RoTHE u. Nachtigall, 1980) and finally died at the age of four. Almost four months previous to the death of the queen, the worker bees built a queen cell which was not used later to rear a queen. At least six other queen cells were constructed by the worker bees and two of them were used to rear queens. Both queens were well developed but only one survived (Fig. 1). To continue brood rearing in the flight-room, a young fertile queen was given to the hive as a substitute for the flight-room queen.

The life span of worker bees was determined three times and the mean value obtained was 39.4 days (Fig. 2). This result agrees with another value also obtained in bees which have been reared in a flightroom (MEYER, 1982). It seems that worker bees live longer in flight-rooms than free-flying summer bees. Pollen feeding could be one reason for the increased life spans of the flight-room bees.

\section{RÉSUMÉ}

ELEVAGE SPONTANÉ DE REINES ET DURÉE DE VIE DES OUVRIÈRES D'ABEILLES EN CHAMBRE DE VOL

Une reine d'abeille a été maintenue pendant près de 2 ans dans une chambre de vol (RoTHE u. NACHTIGALL, 1980) et est morte finalement à l'âge de 4 ans. Environ 4 mois avant sa mort les ouvrières ont construit une cellule royale qui ne fut pas ensuite utilisée pour élever une reine. Six autres cellules royales au moins ont été construites par les ouvrières et deux d'entre elles ont servi à un élevage royal. Les deux 
reines qui en sont nées étaient bien développées mais une seule survécut (Fig. 1). On l'a remplacée par une jeune reine féconde afin que l'élevage de couvain dans la chambre de vol puisse se poursuivre.

On a déterminé la durée de vie des ouvrières par 3 séries de mesures réparties sur les diverses saisons. La valeur moyenne obtenue est de 39,4 jours (Fig. 2). Ce résultat concorde avec une autre valeur obtenue également sur des abeilles en chambre de vol (MEYer, 1982). Il semble que les ouvrières vivent plus longtemps en chambre de vol que les ouvrières d'été en plein air. Le nourrissement au pollen pourrait être une explication à cette durée de vie accrue des ouvrières en chambre de vol.

\section{LITERATUR}

BUtLeR C. G., 1954. - The world of the honeybee. Collins, Saint-James Place, London.

FrisCH K. von, 1969. - Aus dem Leben der Bienen. Springer Verlag, Berlin Heidelberg, N.Y.

Grout R. A. und RutTner F., 1973. - Beute und Biene. Ehrenwirth Verlag München.

Hornsmann E., 1956. - Die Welt der Biene. Kindler Verlag München.

Leuenberger F., 1948. - Der Schweizerische Bienenvater. Lehrbuch der Bienenzucht.

Maurizio A., 1950. - The influence of pollen feeding and brood rearing on the length of life and physiological condition of the honeybee. Bee World, 31 (2), 9-12.

MeYer K., 1982. - Flugraum-Beobachtungen über Sammelverhalten, Pollenvorräte und Brutausfall. Vortrag bei der Arbeitstagung der Arbeitsgemeinschaft der Institute für Bienenforschung E. V., Apidologie, 13 (4).

Peschetz H., 1954. - Vom Anfänger zum Meister, Carnica-Bienenbuch. Verlag Absatia, ColmarFreiburg.

RAUTENBerg H. J., 1956. - Moderne erfolgreiche Bienenzucht. Verlag der Deutschen Bienenzeitung.

RIBBANDS R., 1953. - The behaviour and social life of the honeybee. Bee Research Association Limited, London.

Rothe U. und Nachtigall W., 1980. - Zur Haltung von Apis mellifica in einem Flugraum, Apidologie, 11 (1), 17-24. 